\title{
Screening for Diabetic Retinopathy Using an Automated Diagnostic System Based on Deep Learning: Diagnostic Accuracy Assessment
}

\author{
Sílvia Rêgo ${ }^{a}$ b Marco Dutra-Medeiros $^{c-f}$ Filipe Soares $^{a}$ \\ Matilde Monteiro-Soares ${ }^{g}, \mathrm{~h}$
}

\begin{abstract}
${ }^{a}$ R\&D Department, Fraunhofer Portugal AICOS, Porto, Portugal; ${ }^{b}$ Faculty of Medicine of the University of Porto,

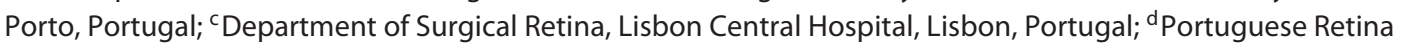
Institute, Lisbon, Portugal; ${ }^{e}$ Chronic Diseases Research Center, NOVA Medical School, NOVA University of Lisbon,

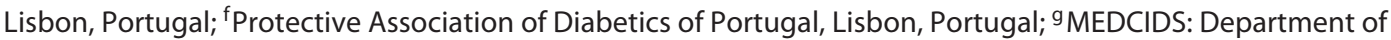
Community Medicine, Health Information and Decision, Faculty of Medicine of the University of Porto, Porto,

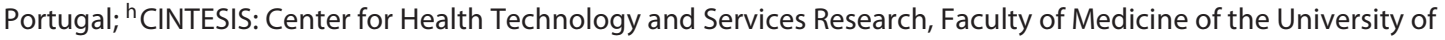
Porto, Porto, Portugal
\end{abstract}

\section{Keywords}

Diabetic retinopathy $\cdot$ Screening $\cdot$ Artificial intelligence .

Automated diagnosis

\begin{abstract}
Purpose: To evaluate the diagnostic accuracy of a diagnostic system software for the automated screening of diabetic retinopathy (DR) on digital colour fundus photographs, the 2019 Convolutional Neural Network (CNN) model with Inception-V3. Methods: In this cross-sectional study, 295 fundus images were analysed by the CNN model and compared to a panel of ophthalmologists. Images were obtained from a dataset acquired within a screening programme. Diagnostic accuracy measures and respective $95 \% \mathrm{Cl}$ were calculated. Results: The sensitivity and specificity of the CNN model in diagnosing referable DR was $81 \%(95 \% \mathrm{Cl} 66-90 \%)$ and 97\% (95\% Cl 95-99\%), respectively. Positive predictive value was $86 \%(95 \% \mathrm{Cl} 72-94 \%)$ and negative predictive value 96\% (95\% Cl 93-98\%). The positive likelihood ratio was 33 (95\% Cl 15-75) and the negative was 0.20 (95\% Cl 0.11-0.35). Its clinical impact is demonstrated by the change observed in the pre-test probability of referable DR (assuming a prevalence of $16 \%$ ) to a post-test probability for a positive test re-
\end{abstract}

karger@karger.com

(c) 2020 S. Karger AG, Basel

www.karger.com/oph

Karger! sult of $86 \%$ and for a negative test result of $4 \%$. Conclusion: A CNN model negative test result safely excludes $D R$, and its use may significantly reduce the burden of ophthalmologists at reading centres.

(c) 2020 S. Karger AG, Basel

\section{Introduction}

Human grading telemedicine-based screening of diabetic retinopathy (DR) is the method of choice in different parts of the world [1] to early identify people with DR, so that they can be effectively treated, and, therefore, avoid vision loss. Regular screening for detection of DR is recommended for all individuals with diabetes [1]. Digital-based colour fundus images are acquired with nonmydriatic retinal cameras operated by a trained eye technician in local settings, usually at primary care offices [1], envisioning a higher rate of patient compliance [2]. Subsequent remote image evaluation by a retina specialist [ 3 , 4] helps to deal with shortage of ophthalmologists [5]. Patients are referred to a retina specialised clinic if clinical signs of severe non-proliferative DR, proliferative DR or moderate macular oedema are shown on retinal photo- 
graphs [6]. This specific condition is commonly named as referral DR. However, telescreening has some potential vulnerabilities, namely the complex and time-consuming task of interpreting eye fundus images for DR diagnosis and the low reliability between ophthalmologists in this task, varying from weak to substantial $(\mathrm{k} 0.19-0.75)[7,8]$.

DR affects $34.6 \%$ of individuals with diabetes [9]. As this number is expected to increase from 382 million in 2013 to 592 million in 2035 [10], the interest in automated methods increased over the last years [11]. Current research includes the estimation of diagnostic accuracy of deep learning-based diagnostic systems [12]. The Convolutional Neural Network (CNN) model with Inception$\mathrm{V} 3$ is an artificial neural network based on deep learning for automated detection of DR [13]. In a previous pilot study to determine preliminary safety and performance with individuals with diabetes referred to ophthalmology because of DR, the software achieved a sensitivity of $74 \%$ and a specificity of 95\% [14]. Nevertheless, this innovative diagnostic test needs to be clinically validated in people with the target disease in similar conditions to those in which the test is intended to be used $[15,16]$. For this purpose, sensitivity, specificity, predictive values and likelihood ratios should be used $[15,16]$. This information is relevant for clinicians who want to apply the findings of the study to decide whether to adopt the test. We conducted a study to evaluate the diagnostic accuracy and clinical usefulness of the software in a larger population of individuals with diabetes, with and without DR, in a screening context.

\section{Methods}

Study Design and Image Selection

A cross-sectional observational study was conducted using anonymised retinal images of individuals with diabetes from EyePACS, a publicly available dataset from primary care offices in the United States of America [17]. Images were non-stereoscopic, acquired by trained camera operators with a variety of non-mydriatic digital retinal cameras, including Canon CR-DGi and Canon CR-1, and with selective pupil dilation $[17,18]$. A study sample of 350 images was randomly selected from the database containing 53,571 images.

Sample size calculation was made for a binary test outcome and the following aspects: an expected sensitivity of at least 70\% [14] (given the result of a preliminary study with the neural network and also because this value is considered to be the lowest value accepted for screening tools [19]), a confidence level of 95\%, a type I error of $5 \%$, a power of $95 \%$, a type II error of $12 \%$, and a prevalence of DR of $20 \%$ [20]. Accordingly, the required sample size was composed of 286 images. Considering a median of image rejection rate of $18.3 \%$ [3] for fundus images because they were ungradable, the sample size was estimated as 350 images.

Diabetic Retinopathy Automated Screening
Index Test

The 2019 CNN model with Inception-V3 in EyePACS data is an automated diagnostic system based on Deep Learning trained in EyePACS and Messidor datasets that automatically identifies DR in digital colour fundus images [13]. It provides a dichotomised classification as presence or absence of DR or referable DR. The process of image analysis is fully automatic and was performed on a computer.

\section{Reference Standard}

The reference standard was established by taking the majority decision from 3 independent graders: the eye care specialist of the EyePACS, one general ophthalmologist and one retina specialist. They used a modified version of the International Clinical Disease Severity Scale (ICDSS) for DR, that was derived from the Early Treatment of Diabetic Retinopathy Study (ETDRS) and is used in most DR screenings [21]. Accordingly, DR was classified into 4 severity levels: without retinopathy $(\mathrm{R} 0)$, mild non-proliferative $(\mathrm{R} 1)$, moderate or severe pre-proliferative (R2) and proliferative (R3). Diabetic macular oedema was classified as M1. Images deemed not classifiable by at least one grader were not included in the study. Images graded as R0 were classified as negative for the presence of DR and images graded as R1, R2 or R3 or M1 as positive for DR; R0 and R1 were classified as negative for the presence of referable $\mathrm{DR}$ and $\mathrm{R} 2, \mathrm{R} 3$ or $\mathrm{M} 1$ as positive.

Analysis

The software results were compared to those of the clinical reference standard. Sensitivity, specificity, positive predictive value (PPV) and negative predictive value (NPV), with 95\% CI were calculated as measures of diagnostic accuracy in accordance with the STARD 2015 guidelines for reporting diagnostic accuracy studies [22]. Sensitivity is the proportion of images identified with the disease in those with a positive test result, and specificity is the proportion of images identified without the disease in those with a negative test result. PPV is the proportion of images with a positive result that have the disease, and NPV is the proportion of images with a negative result that do not have the disease.

The likelihood ratios were also calculated because they give an estimation of how likely it is for an individual with diabetes to have $\mathrm{DR} /$ referable DR, thereby helping clinical decision-making [16]. The positive likelihood ratio (LR+) of a test corresponds to a ratio between the proportion of true positives (i.e., sensitivity) and the proportion of false positives (i.e., 1 - specificity) [16]. In contrast, the negative likelihood ratio (LR-) of a test corresponds to a ratio between the proportion of false negatives (i.e., 1 - sensitivity) and the proportion of true negatives (i.e., specificity) [16].

The clinical usefulness of the software test was assessed through the extent to which it helps to modify the pre-test probability of occurrence of DR/referable DR in individuals with diabetes [16]. The post-test probability was calculated using the graphical tool Fagan's nomogram, knowing the pre-test probability (i.e., the prevalence of disease in the study population) and the likelihood ratios [16].

\section{Results}

Of the 350 fundus images, 55 (16\%) were classified as ungradable by at least one of the ophthalmologists and were excluded. All classifiable images had software automated classification as described in Figure 1.

Ophthalmologica 2021;244:250-257 


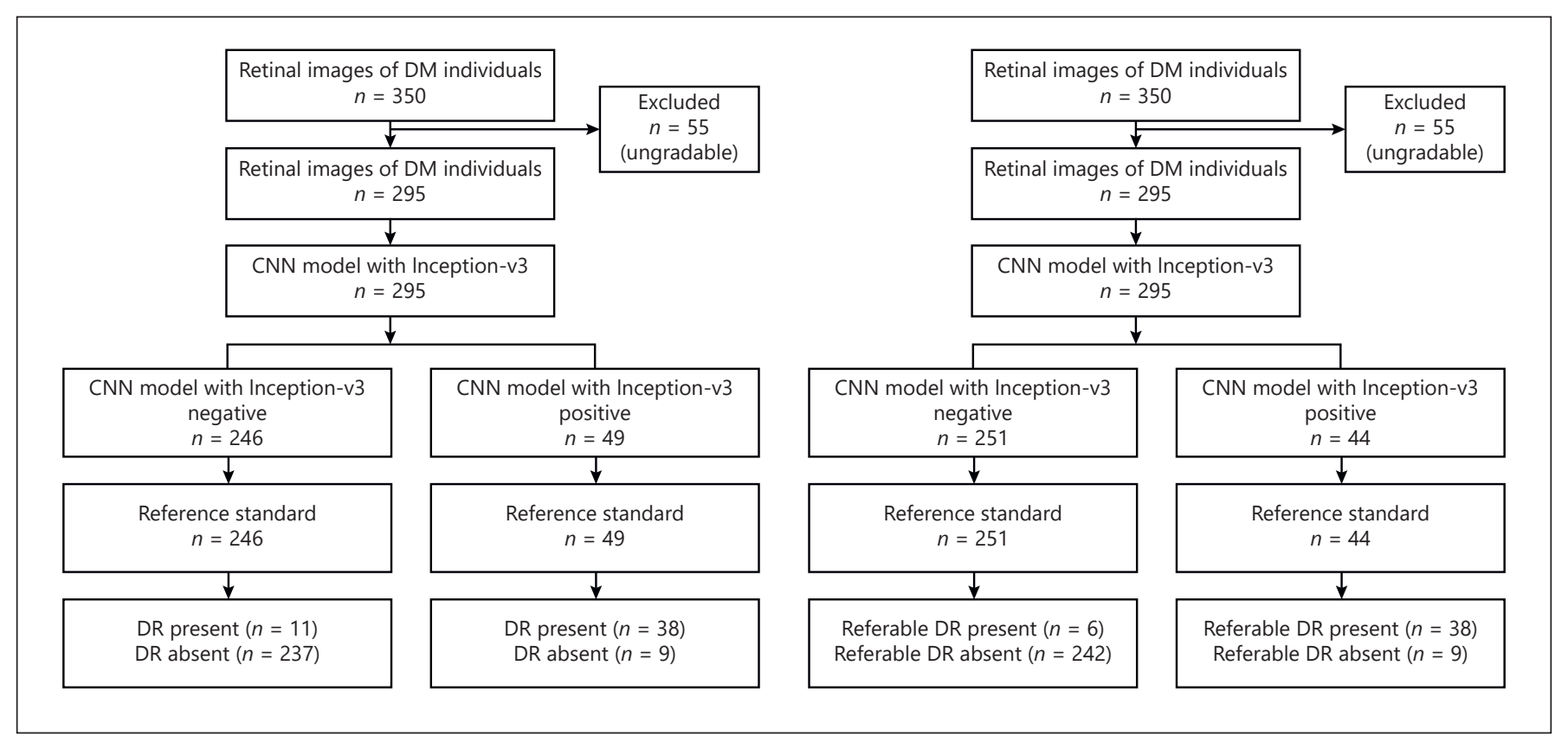

Fig. 1. Flow of participants and tests results. DR, diabetic retinopathy; DM, diabetes mellitus.

Table 1. Diagnosis provided by the CNN model with Inception-V3 compared to the reference standard

\begin{tabular}{lrrr}
\hline \multirow{2}{*}{$\begin{array}{l}\text { CNN model with } \\
\text { Inception-V3 }\end{array}$} & \multicolumn{2}{l}{ Human grading } & \multirow{2}{*}{ Total } \\
\cline { 2 - 3 } & DR present & DR absent & \\
\hline DR present, $n(\%)$ & $38(77.6)$ & $11(22.4)$ & $49(16.7)$ \\
DR absent, $n(\%)$ & $9(3.70)$ & $237(96.3)$ & $246(83.4)$ \\
Total, $n(\%)$ & $47(15.9)$ & $248(84.1)$ & $295(100)$ \\
\hline RDR present, $n(\%)$ & $38(86.4)$ & $6(2.42)$ & $44(14.9)$ \\
RDR absent, $n(\%)$ & $9(3.58)$ & $242(96.4)$ & $251(85.1)$ \\
Total, $n(\%)$ & $47(15.9)$ & $248(84.1)$ & $295(100)$ \\
\hline
\end{tabular}

DR, diabetic retinopathy; RDR, referable diabetic retinopathy.

The prevalence of DR was $15.9 \%$ (95\% CI 12.0-20.7\%). The software correctly identified 38 of the $47(81 \%)$ analysable images with any DR and 38 of $47(81 \%)$ with referable DR. Among the 248 analysable images with no DR according to the panel of ophthalmologists, there were 237 (96\%) and 242 (98\%) images with DR absent output for any DR and referable DR, respectively. There were 11 false positives and 9 false negatives for any DR, and 6 false positives and 9 false negatives for referable DR. The software and the reference standard classification for all 295 images are described in Table 1.
Table 2. CNN model with Inception-V3 diagnostic accuracy for DR when compared to ophthalmologist's classification

\begin{tabular}{lcc}
\hline $\begin{array}{l}\text { CNN model with } \\
\text { Inception-V3 }\end{array}$ & DR (95\% CI) & RDR (95\% CI) \\
\hline Sensitivity, \% & $80.8(66.3-90.4)$ & $80.8(66.3-90.3)$ \\
Specificity, \% & $95.6(92.0-97.6)$ & $97.3(94.6-99.0)$ \\
PPV, \% & $77.6(63.0-87.8)$ & $86.4(72.0-94.3)$ \\
NPV, \% & $96.3(92.9-98.2)$ & $96.4(93.1-98.2)$ \\
LR+ & $18.2(10.1-33.0)$ & $33.4(15.0-74.6)$ \\
LR- & $0.200(0.111-0.361)$ & $0.196(0.109-0.353)$ \\
\hline
\end{tabular}

PPV, positive predictive value; NPV, negative predictive value; LR+, positive likelihood ratio; LR-, negative likelihood ratio; DR, diabetic retinopathy; RDR, referable diabetic retinopathy.

Forty-seven images had DR according to ophthalmologists grading, and sensitivity and specificity of the software in identifying DR were of 80.8 and $95.6 \%$, respectively, with PPV and NPV of 77.6 and $96.3 \%$, respectively. Although some of the values were higher when the clinical outcome was referable DR, no significant statistical differences were found in the observed accuracy measures between the identification of DR or referable DR. Table 2 shows the calculated sensitivity, specificity, PPV, NPV, LR+ and LR- for the software for screening of DR and referable DR with respective 95\% CI. 


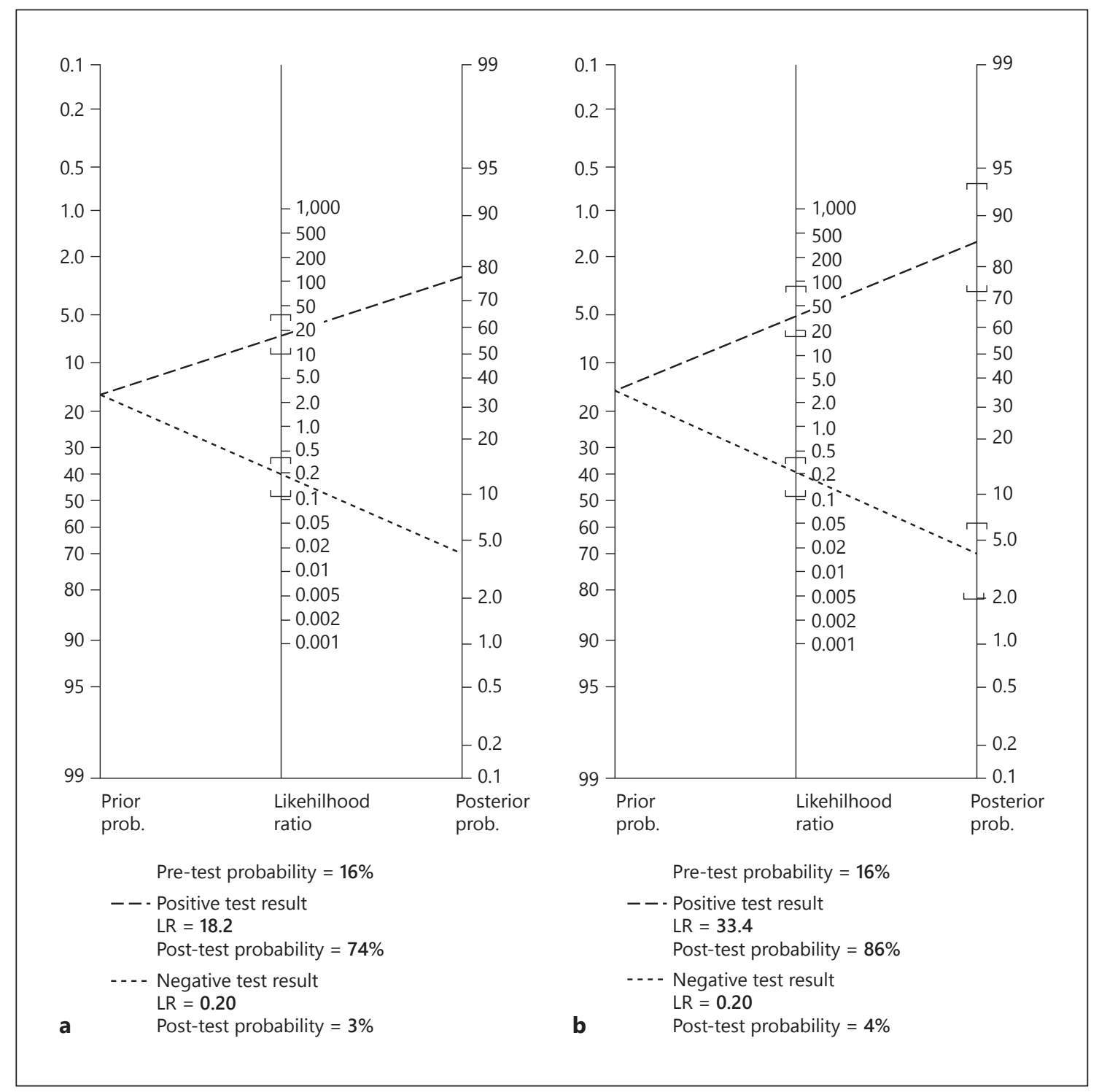

Fig. 2. Fagan's nomogram. a Diabetic retinopathy: pre-test probability $=16 \%$. A positive test result leads to an increase in the post-test probability to $74 \%$. A negative test result leads to a decrease in the post-test probability to $3 \%$. b Referable diabetic retinopathy: pre-test probability $=16 \%$. A positive test result leads to an increase in the post-test probability to $86 \%$. A negative test result leads to a decrease in the post-test probability to $4 \%$.

For DR, the LR+ was of 18.2, while the LR- was 0.20 . When using Fagan's nomogram, a straight line from a patient's pre-test probability (16\%) is drawn through the LR for a positive and for a negative test result, which points to the post-test probability of DR for both cases. This led to a post-test probability for a positive test result of $74 \%$ and a post-test probability for a negative test result of 3\%, as shown in Figure 2. The absolute difference between pre- and post-test probabilities of DR is 0.58 for positive and 0.13 for negative test results. Similarly, for referable DR, the LR+ was of 33, while the LR- was of 0.20 . Given an estimated prevalence of $16 \%$, if the patient tests positive, the post-test probability that he/she has truly referable DR would be $86 \%$; if the patient tests negative, the post-test probability that he/she has truly referable DR would be $4 \%$. The absolute difference between preand post-test probabilities of referable DR is 0.70 for positive and 0.12 for negative test results. 


\section{Discussion}

Diagnostic automation for DR using artificial intelligence will likely become essential as our rapidly ageing societies and increasing trends in diabetes prevalence continue to challenge healthcare systems with more demand for observation of fundus images. With the clinical use of the CNN model with Inception-V3 automated diagnostic system for DR, we can expect 85 fewer observations by ophthalmologists at reading centres, at a $<4 \%$ probability of missing referable DR. The use of the $\mathrm{CNN}$ model for DR referral triage classification would safely enable to reduce ophthalmologists' workload. If we were to refer all images automatically classified as DR, this would result in a slightly higher number of observations needed when compared with sending referable DR only (reduction of 83 vs. 85\%) without an important impact on the proportion of false negatives. Therefore, we consider that the best artificial intelligence screening approach would be for ophthalmologists at the reading centre to receive images classified as referable DR-positives by the software. In this way, clinical practices could easily accommodate the expected increase in the number of required screenings worldwide. If this software is used at point-of-care at the time of image acquisition, it has the additional advantage of immediate communication of test results to the patient and more rapid observation of screen-positive patients by an ophthalmologist.

The CNN model with Inception-V3 demonstrated an $81 \%$ sensitivity for the detection of referable DR, which is considered to be safe according to the NHS' Exeter Standard, being above the established $80 \%$ as the minimum sensitivity [23]. Only 1 in 10 patients with referable DR (4\% of 38 patients) was missed by the software. Also, with a specificity of $98 \%$ for referable DR diagnosis, it exceeded the recommended value of $95 \%$ [23], showing that it correctly excludes people without the disease with $2 \%$ of false positives (6 in 248). This is an important attribute as the individuals screened positive will be advised to go to a retina specialised clinic, which represents a waste of resources. Moreover, the high specificity enables us to conclude that if a person tests positive with the software, it rules in referable DR. Values of sensitivity and specificity observed for DR were similar to the ones obtained for referable DR.

Several studies report sensitivities that vary from 87 to $97 \%$ and specificities from 59 to $98 \%$ [24]. A recent metaanalysis showed that deep learning algorithms perform well in screening for DR, having a pooled area under the receiving operating curve of 0.97 (95\% CI 0.95-0.98), and a pooled sensitivity and specificity of $83 \%$ (95\% CI $83-$ $83 \%$ ) and $92 \%$ (95\% CI 92-92\%), respectively, for detecting referable DR from fundus images, with additional reduction in misdiagnosis [25]. Studies reporting diagnostic accuracy measures of other deep learning algorithms, in the same population and using the EyePACS dataset, have reported sensitivities that ranged from 30 to $100 \%$ and from 82 to $92 \%$ for DR and referable DR, respectively, and specificities that varied from 85 and $99 \%$ and from 71 to $97 \%$ for DR and referable DR, respectively $[25,26]$. When compared to the EyeArt, which showed a sensitivity and a specificity of $91 \%$ (95\% CI 90.9-91.7\%) for referable DR detection, the CNN model with Inception-V3 had statistically lower sensitivity, but statistically higher specificity. The IDx-DR artificial intelligence diagnostic system, clinically validated in the same population, had similar values of both sensitivity and specificity to the $\mathrm{CNN}$ model with Inception-V3, with a sensitivity of $87 \%$ and a specificity of $90 \%$, therefore both higher than the minimum set by the FDA: 85 and $82.5 \%$ for sensitivity and specificity, respectively [27]. Deep learning algorithms have also been applied recently to retinal optical coherence tomography (OCT) and OCT angiography (OCTA) images for automated diagnosis of DR, because these imaging techniques provide higher sensitivity to detect early diabetic retinal changes [28]. Diagnostic accuracy studies have not been published yet, but preliminary internal validation tests show comparable diagnostic accuracy to the one using fundus images: Sandhu et al. [29] reported their algorithm to have sensitivity of $93 \%$ and specificity of $95 \%$, and the work of Li et al. [30] achieved a sensitivity of $90 \%$ and specificity of $95 \%$; using OCTA images, sensitivity of $98 \%$ and specificity of $87 \%$ were reported by Sandhu et al. [31], and the system of Le et al. [32] achieved $84 \%$ sensitivity and $91 \%$ specificity.

One measure that can specifically help the clinician to estimate the probability of an individual to have the disease is the predictive values [16]. A high NPV was achieved with the software $-96 \%$, indicating the probability for a patient not to have referable DR given that the test result is negative. This value gives very high certainty to safely exclude referable DR in subjects screened negative with the CNN model with Inception-V3 and thus they do not need to be sent to a medical appointment with an ophthalmologist. A PPV of $86 \%$ reveals the probability for a positive test result to indicate the presence of referable $\mathrm{DR}$, which is a good value in screening settings, where prevalence of the disease is low, making the PPV decrease [33]. To the best of our knowledge, only 2 other artificial intelligence systems described predictive values: the $\mathrm{CNN}$ 
model presented higher PPV (86vs. 72\%) than the EyeArt, and slightly lower NPV (96 vs. 98\%), despite EyeArt's values were under our CIs; the study of Kanagasingam et al. [34] reported an NPV of $100 \%$, but a PPV of only $12 \%$ (95\% CI 8-18\%), which is significatively lower compared to the $\mathrm{CNN}$ model and demonstrating high false-positive rate, despite the high specificity of $92 \%$ (95\% CI 87-96\%) that is not significatively higher than the CNN model's specificity.

When a clinician needs to interpret the test result for the individual patient in a different population, likelihood ratios, which are independent of prevalence and inherent to the test, are advantageous. A diagnostic test will be more useful to the extent that its positive likelihood ratio is of greater magnitude and that its negative likelihood ratio is smaller. In our study, for referable DR, the positive likelihood ratio was of 33 and negative likelihood ratio 0.20 . Both values are statistically significantly higher than the ones reported in a systematic review: 14.11 (95\% CI 9.91-20.07), and 0.10 (95\% CI 0.07-0.16), for positive and negative likelihood ratios, respectively [25]. Because clinicians are more familiar with thinking in terms of probabilities, using Fagan's nomogram to translate likelihood ratios into post-test probabilities, the result showed that after the $\mathrm{CNN}$ model with Inception-V3 positive result, the probability of a patient having referable DR has increased from 16 to $86 \%$. This probability is high and indicates that referable DR is likely $[35,36]$ and, therefore, observation by an ophthalmologist is recommended. This value is not very high though, which is not surprising, since false positives are expected in screening. Considering the recommendation that a clinician should be at least $90 \%$ certain to make a definite diagnosis [37], and that the software has screening purposes, $86 \%$ seems to provide high certainty in relation to the presence of disease and adequateness in sending the patient for diagnosis confirmation in further observation. Likewise, with a negative $\mathrm{CNN}$ model with Inception-V3 result, the probability of referable DR shifts from 20 to $4 \%$, and the disease can be excluded with reasonable certainty.

The CNN model with Inception-V3 demonstrated a good performance regarding diagnostic accuracy as an artificial intelligence screening test for referable DR, identifying and excluding disease with a good balance, enabling few both false positives and negatives. Predictive values, likelihood ratios and the post-test probability of disease showed that the software supports clinicians in determining a patient's care pathway in screening of referable DR, helping to estimate the probability of an individual patient having or not having the disease. As such, all measures of diagnostic accuracy converge to demonstrate the adequacy and clinical usefulness of the CNN model with Inception-V3, with safety in screening decisions about individual people and advantages for healthcare systems.

A limitation of our study was that the reference standard panel was not exclusively composed of retina specialists, as one was a general ophthalmologist. Literature shows lower levels of agreement between general ophthalmologists when compared to the agreement between retina specialists $[7,8]$. Additionally, the DR prevalence of the study population (16\%) was lower than the value used for sample size calculation (20\%); these could have affected the power of the test, with wider CIs and subsequent decrease in precision. However, the CI for prevalence includes the prevalence used for the sample size calculation. On the other hand, we assumed a sensitivity of $70 \%$ for sample size calculation, having taken into account a previous study [14] with an old version of the algorithm and conducted with patients with DR screenpositives only, which may have led to a higher precision.

Further work is needed to validate the CNN model with Inception-V3, envisioning its implementation in clinical practice, namely, through the conduction of a multicentre study with patients with diabetes followed at primary care institutions, comparing its results against a panel of retina specialists as reference standard. Assessment of its effectiveness in real-world clinical settings is also desirable. Future research should consider investigating the diagnostic accuracy of the software on retinal images acquired with different fundus cameras.

\section{Conclusion}

This study analysed the properties of the 2019 CNN model with Inception-V3 artificial intelligence-based automated classification of single-field digital eye fundus images acquired with table-top fundus cameras for referable DR screening. We concluded that the CNN model based on Deep Learning compared favourably with the reference standard and met the pre-established values of the Food and Drug Administration and the NHS' Exeter Standard. Therefore, this diagnostic test accuracy study provides evidence that the software correctly identifies and rules out DR and justifies a subsequent multicentre prospective diagnostic accuracy study with an independent cohort of individuals with diabetes to enable generalization of results. Considering the clinical application of this diagnostic test for screening, it gives the clinician 
strong evidence to support ruling out referable DR in images classified as negative, with few false negatives. Our results suggest that the integration of the $\mathrm{CNN}$ model with Inception-V3 into the DR screening workflow shows promise in alleviating the great demanding and timeconsuming task of ophthalmologists at reading centres.

\section{Acknowledgement}

The authors acknowledge Telmo Barbosa, MSc, Fraunhofer Portugal AICOS, for the development and management of the web annotation tool for classification of retinal images by ophthalmologists, and Tânia Borges, MD, MSc, and Gustavo Bacelar-Silva, MD, MSC, for providing image classifications. We acknowledge João Gonçalves, MSc, Fraunhofer Portugal AICOS, for providing the CNN model with Inception-V3 outputs, Ricardo Graça, MSc, Fraunhofer Portugal AICOS, for inputs on data preparation, and Ana Correia de Barros, PhD, Fraunhofer Portugal AICOS, for grammatical and stylistic revision of the manuscript. We also acknowledge Kaggle Inc. (https://www.kaggle.com/c/diabetic-retinopathy-detection/data) and EyePACS, LLC (http://www.eyepacs. com) for providing the eye fundus images dataset used in this study.

\section{Statement of Ethics}

Ethical approval was not required or obtained for this study, because we used a database of retinal images collected by EyePACS, LLC and publicly available through Kaggle Inc.

\section{Conflict of Interest Statement}

S.R. and F.S. are employees of Fraunhofer Portugal AICOS, Porto, Portugal, and this institution is developing a decision-support system for diabetic retinopathy automatic classification. M.M.-S. and M.D.-M. have no financial involvement with Fraunhofer Portugal AICOS and no competing interests. No other relationships or activities could appear to have influenced the submitted work.

\section{Funding Sources}

This work was supported by Fraunhofer Portugal AICOS (Porto, Portugal); the development of the web-platform for classification of retinal images was supported by Project MDevNet - National Network for Transfer of Knowledge of Medical Devices, in the scope of the Portuguese national programme NORTE 2020 under Portugal 2020. The sponsor or funding organization had no role in the design or conduct of this research.

\section{Author Contributions}

S.R. conception and design, acquisition, analysis and interpretation of data, article draft, final approval of the version to be published, agreement to be accountable for all aspects of the work. M.D.-M. interpretation of data, article revision, final approval of the version to be published, agreement to be accountable for all aspects of the work. F.S. conception and design, acquisition and interpretation of data, article revision, final approval of the version to be published, agreement to be accountable for all aspects of the work. M.M.-S. conception and design, interpretation of data, article revision, final approval of the version to be published, agreement to be accountable for all aspects of the work.

\section{References}

1 World Health Organization. Prevention of blindness from diabetes mellitus. Report of a WHO Consultation, Switzerland, November 2005.

2 Lewis K. Improving patient compliance with diabetic retinopathy screening and treatment. Community Eye Health. 2015;28(92):68-9.

3 Browning DJ. Screening for Diabetic Retinopathy. In: Diabetic Retinopathy: EvidenceBased Management. New York: SpringerVerlag; 2010. p. 369-385.

4 Das T, Raman R, Ramasamy K, Rani PK. Telemedicine in diabetic retinopathy: current status and future directions. Middle East Afr J Ophthalmol. 2015 Apr-Jun;22(2):174-8.

5 Pieczynski J and Grzybowski A. Review of Diabetic Retinopathy Screening Methods and Programmes Adopted in Different Parts of the World. Eur Ophthalmic Rev. 2015; 09(01): 49.

6 Diabetic retinopathy (DR): management and referral. Community Eye Health. 2015; 28(92):70-1.
7 Ruamviboonsuk P, Teerasuwanajak K, Tiensuwan M, Yuttitham K; Thai Screening for Diabetic Retinopathy Study Group. Interobserver agreement in the interpretation of single-field digital fundus images for diabetic retinopathy screening. Ophthalmology. 2006 May;113(5):826-32.

8 Gegundez-Arias ME, Ortega C, Garrido J, Ponte B, Alvarez, F, Marin D. Inter-observer Reliability and Agreement Study on Early Diagnosis of Diabetic Retinopathy and Diabetic Macular Edema Risk. In: 4th International Conference on Bioinformatics and Biomedical Engineering, IWBBIO (ed Ortuño F, Rojas I), Granada, Spain, 20-22 April 2016, pp 36979.

9 Yau JWY, Rogers SL, Kawasaki R et al. Global Prevalence and Major Risk Factors of Diabetic Retinopathy. Diabetes Care. 2012 Mar; 35(3):556-64.

10 Guariguata L, Whiting DR, Hambleton I, Beagley J, Linnenkamp U, Shaw JE. Global estimates of diabetes prevalence for 2013 and projections for 2035. Diabetes Res Clin Pract. 2014 Feb;103(2):137-49.

11 Korot E, Wood E, Weiner A, Sim DA, Trese M. A renaissance of teleophthalmology through artificial intelligence. Eye (Lond). 2019 Jun;33(6):861-3.

12 Ting DS, Pasquale LR, Peng L, Campbell JP, Lee AY, Raman R, et al. Artificial intelligence and deep learning in ophthalmology. $\mathrm{Br} \mathrm{J}$ Ophthalmol. 2019 Feb;103(2):167-75.

13 Gonçalves J, Conceição T, Soares F. Inter-observer Reliability in Computer-aided Diagnosis of Diabetic Retinopathy. In: Proceedings of the 12th International Joint Conference on Biomedical Engineering Systems and Technologies - Volume 5 HEALTHINF, Prague, Czech Republic, February 22-24, 2019, p. 481-491.

14 Felgueiras S, Costa J, Gonçalves J, Soares F. Mobile-based Risk Assessment of Diabetic Retinopathy using a Smartphone and Adapted Ophthalmoscope. HEALTHINF, Funchal, Madeira, Portugal, January 19-21, 2018, p. 168-175. 
15 Cerda LJ, Cifuentes AL. Clinical use of diagnostic tests (Part 1): Analysis of the properties of a diagnostic test. Rev Chil Infectologia Organo Of Soc Chil Infectologia. 2010 Jun;27(3): 205-8.

16 Cifuentes L, Cerda J. Clinical use of diagnostic tests (Part 2). Clinical application and usefulness of a diagnostic test. Rev Chil Infectologia Organo Of Soc Chil Infectologia. 2010 Aug; 27(4):316-9.

17 EyePACS [Internet]. Clinical Services for Diabetic Retinopathy [cited 2020 July 20]. Available from: https://www.eyepacs.com/.

18 Cuadros J and Sim I. EyePACS: an open source clinical communication system for eye care. Stud Health Technol Inform. 2004; 107(Pt 1):207-11.

19 Bujang MA, Adnan TH. Requirements for Minimum Sample Size for Sensitivity and Specificity Analysis. J Clin Diagn Res. 2016 Oct;10(10):YE01-06

20 Cuadros JA. Telemedicine-based diabetic retinopathy screening programs: an evaluation of utility and cost-effectiveness. Smart Homecare Technol Telehealth. 2015;3:11927.

21 Wu L, Fernandez-Loaiza P, Sauma J, Hernandez-Bogantes E, Masis M. Classification of diabetic retinopathy and diabetic macular edema. World J Diabetes. 2013 Dec;4(6):290_ 4.

22 Cohen JF, Korevaar DA, Altman DG, Bruns DE, Gatsonis CA, Hooft L, et al. STARD 2015 guidelines for reporting diagnostic accuracy studies: explanation and elaboration. BMJ Open 2016;6 (11):e012799.
23 Scanlon PH. The English National Screening Programme for diabetic retinopathy 2003 2016. Acta Diabetol. 2017 Jun;54(6):515-25.

24 Raman R, Srinivasan S, Virmani S, Sivaprasad S, Rao C, Rajalakshmi R. Fundus photographbased deep learning algorithms in detecting diabetic retinopathy. Eye (Lond). 2019 Jan; 33(1):97-109.

25 Islam MM, Yang HC, Poly TN, Jian WS, Jack Li YC. Deep learning algorithms for detection of diabetic retinopathy in retinal fundus photographs: A systematic review and meta-analysis. Comput Methods Programs Biomed. 2020 Jul;191:105320.

26 Bhaskaranand M, Ramachandra C, Bhat S, Cuadros J, Nittala MG, Sadda SR, et al. The Value of Automated Diabetic Retinopathy Screening with the EyeArt System: A Study of More Than 100,000 Consecutive Encounters from People with Diabetes. Diabetes Technol Ther. 2019 Nov;21(11):635-43.

27 Abràmoff $\mathrm{MD}$, Lavin $\mathrm{PT}$, Birch $\mathrm{M}$, Shah $\mathrm{N}$, Folk JC. Pivotal trial of an autonomous AIbased diagnostic system for detection of diabetic retinopathy in primary care offices. NPJ Digit Med. 2018 Aug;1(1):39.

28 Optometry Times [Internet]. OCT, OCTA show promise in screening for DR [cited 2020 August 28]. Available from: https://www.optometrytimes.com/view/oct-octa-showpromise-in-screening-for-dr.

29 Sandhu HS, Eltanboly A, Shalaby A, Keynton RS, Schaal S, El-Baz A. Automated Diagnosis and Grading of Diabetic Retinopathy Using Optical Coherence Tomography. Invest Ophthalmol Vis Sci. 2018 Jun;59(7):3155-60.
30 Li X, Shen L, Shen M, Tan F, Qiu CS. Deep learning based early stage diabetic retinopathy detection using optical coherence tomography. Neurocomputing. 2019 Dec;369:13444.

31 Sandhu HS, Eladawi N, Elmogy M, Keynton R, Helmy O, Schaal S, et al. Automated diabetic retinopathy detection using optical coherence tomography angiography: a pilot study. Br J Ophthalmol. 2018 Nov;102(11): 1564-9.

32 Le D, Alam M, Yao CK, Lim JI, Hsieh YT, Chan RV, et al. Transfer Learning for Automated OCTA Detection of Diabetic Retinopathy. Transl Vis Sci Technol. 2020 Jul;9(2): 35-35.

33 Šimundić AM. Measures of Diagnostic Accuracy: basic Definitions. EJIFCC. 2009 Jan; 19(4):203-11.

34 Kanagasingam Y, Xiao D, Vignarajan J, Preetham A, Tay-Kearney M, Mehrotra A. Evaluation of Artificial Intelligence-Based Grading of Diabetic Retinopathy in Primary Care. JAMA Netw Open 2018;1(5):e182665.

35 Fritz JM, Wainner RS. Examining diagnostic tests: an evidence-based perspective. Phys Ther. 2001 Sep;81(9):1546-64.

36 McGee S. Simplifying Likelihood Ratios. J Gen Intern Med. 2002 Aug;17(8):647-650.

37 Parikh R, Parikh S, Arun E, Thomas R. Likelihood ratios: clinical application in day-to-day practice. Indian J Ophthalmol. 2009 May-Jun; 57(3):217-21. 